\title{
Löwer, Wolfgang: Rechtsfragen der Einführung eines Curricularwertes
}

\author{
Wissenschaftsrecht Beiheft 20, VIII, 110 Seiten, Mohr Siebeck Verlag, \\ Thübingen, 2010. Brosch, EUR 34,00. ISBN 978-3-16-150566-9
}

\begin{abstract}
„Das Freiheitsversprechen, dass jeder Zugangsberechtigte einen Studienplatz im Studiengang seiner Wahl am gewünschten Standort erhalten soll, ist in der Geschichte der Bundesrepublik wohl nie vollständig erfüllt worden. Die Nachfrage hat das Platzangebot an konkreten Studienorten in bestimmten Studiengängen regelmäßig überstiegen, so dass das Hochschulzulassungsrecht zum Referenzgebiet der Mangelverwaltung geworden ist". Diese einleitenden Worte Löwers haben für Österreich fast utopische Bedeutung - überlegt bzw fordert man in Österreich doch gerade, den Weg des offenen Hochschulzugangs in Österreich zu verlassen. Von den Universitäten wird die Fixierung einer bestimmten Kapazität von Studienplätzen bzw eine „kapazitätsorientierte" Zulassung gefordert. Nicht nur, weil auf Grund von steigenden Studierendenzahlen in Österreich eine Verschlechterung der Betreuungsqualität befürchtet wird, sondern auch, weil durch politische Entscheidungen in Deutschland (doppelte Abiturienten- bzw Abiturientinnenjahrgänge und die Aussetzung der Wehrpflicht) 100.000 Studierende mehr in Deutschland erwartet werden, so dass zu erwarten ist, dass diese verstärkt nach Österreich drängen werden. Die zitierte „Mangelverwaltung“ wird also noch stärker als bisher direkte Auswirkungen auf Österreich haben und könnte in Österreich $\mathrm{zu}$ einer Änderung der Zugangspolitik führen. Umso interessanter ist in einer solchen Situation den Blick über die Grenzen zu wagen und sich dafür zu interessieren, wie der Nachbar mit diesem Problem umgeht.
\end{abstract}

Die vorliegende Studie Löwers wurde im Auftrag des Ministeriums für Innovation, Wissenschaft, Forschung und Technologie des Landes Nordrhein-Westfalen erstellt und begutachtet aus rechtlicher Sicht einen Neuregelungsvorschlag, der es den Universitäten ermöglichen soll, flexiblere Kapazitätsgrenzen innerhalb einer Bandbreite festzulegen.

Für Österreich sind die Überlegungen - obwohl sie sich auf die deutsche Rechtslage beziehen einerseits deshalb interessant, weil sie eine Auseinandersetzung mit verschiedenen Modellen von Zugangsbeschränkungen bieten, andererseits, weil manche rechtlichen Probleme sich auch in Österreich stellen könnten - wenn auch im Detail unterschiedlich, so doch von den Ansätzen her ähnlich - beispielsweise die Frage des Verhältnisses solcher Regelungen zu Zielvereinbarungen. Gerade im Hinblick auf die derzeit laufenden politischen Diskussionen ist das vorliegende Beiheft für alle an der Thematik Interessierten eine empfehlenswerte Lektüre. 